This is a post-peer-review, pre-copyedit version of an article published in Current Biology. The final authenticated version is available online at: $\mathrm{https} / / / d o i . o r g / 10.1016 / / . c u b .2021 .01 .090$

\title{
The role of scientists in policy making for more sustainable agriculture
}

\author{
Oana Dima ${ }^{1,2}$ and Dirk Inzé1,2* \\ ${ }^{1}$ Department of Plant Biotechnology and Bioinformatics, Ghent University, 9052 Ghent, Belgium. \\ 2 VIB Center for Plant Systems Biology, 9052 Ghent, Belgium. \\ *E-mail: dirk.inze@psb.vib-ugent.be
}

Feeding the growing world population under changing climate conditions, environmental degradation and the recent COVID-19 pandemic poses globally unprecedented challenges on crop production systems that comprise many aspects, such as inputs, management practices, plant protection, soil management and crop varieties. Breeders, geneticists and biotechnologists focus mainly on improving plant varieties through breeding. Since the discovery of Gregor Mendel's laws of inheritance in 1865, plant breeding has undergone many technological breakthroughs, ranging from the ability to make crosses with wild relatives; to mutation breeding since 1920, which increases the rate of genetic variation; and ultimately to modern crop improvement, using technological advances in molecular biology, of which genome editing is the most recent innovation. As of 2012, genome editing by CRISPR became a game-changing new tool for our understanding of the genetic basis of biological processes. In 2020, the Nobel Prize in Chemistry was awarded to Emmanuelle Charpentier and Jennifer A. Doudna for the development of a genome-editing method using CRISPR ${ }^{1}$, highlighting its revolutionary impact. For applications in agriculture, CRISPR emerged as a revolutionary breeding technology that enables the very precise, efficient and cost-effective selection of improved crops (Box 1). Unfortunately, the current European legislation subjects crop varieties obtained using CRISPR under strict GMO regulations, which de facto blocks the introduction of genomeedited crops to the EU market. Not surprisingly, the scientific community has been raising concerns on the current EU status of crops obtained through new genomic techniques, which is based on outdated EU legislation that no longer reflects the current state of scientific evidence and progress. The European Sustainable Agriculture through Genome Editing (EU-SAGE) network ${ }^{2}$, representing researchers from 133 leading European plant science institutes and learned societies, is convinced that Europe needs to enable application of genome editing through developing science-based policies. Recently, a report entitled "Genome editing for crop improvement" has been published by ALLEA (All European Academies), in which EU-SAGE was introduced ${ }^{3}$. The report presents the state-of-the-art of scientific evidence and explores paths to harmonize EU legislation with recent scientific developments. Here, we elaborate on how Europe is lagging behind in embracing the potential of genome editing, and we highlight how scientists can contribute to advising on effective science-based policies for more sustainable agriculture through genome editing. 


\section{Plant science and crop improvement}

Over the last century, plant breeding has become much more knowledge-based and has accelerated the development of improved crop varieties. However, conventional practices in plant breeding will not suffice to reach, in a short time frame, more sustainable plant productivity. It takes many years to develop and improve crops by classical or mutation breeding - time that, in view of rapid climate change, is not available to safeguard global food security. Fortunately, genome editing by CRISPR enables us to develop desired plant varieties in a faster, more efficient and directed manner. One prerequisite for this is that the target genes in the recipient plant are known. In recent years, technological advances in next-generation sequencing and molecular biology have greatly improved our knowledge of the genomes of a plethora of crops. Also, genome editing, by its ease to make changes in the genetic code, has been contributing in an exponential manner to the elucidation of the genetic networks that control all kinds of yield-related traits in both model plants and crops. There are many published examples that demonstrate the potential of genome editing to improve crop yield and quality, as well as to render agriculture more sustainable and climate resilient. The first genome-edited crops have already entered the market ${ }^{4}$, and many more are in the process of commercialization. The Animal and Plant Health Inspection Service (APHIS) of the United States Department of Agriculture (USDA) provided an inquiry process - "Am I regulated?" - to evaluate the regulatory status of genome-edited crops in the US ${ }^{5}$. In total, 168 inquiries were submitted (status as of September 2020), covering many applications in crops. In contrast to Europe, the majority of countries across the world have decided not to regulate genome-edited crops in any way differently from other plant varieties. Remarkably, shortly after Brexit, the UK's Department for Environment Food \& Rural Affairs (DEFRA) launched a consultation on the regulation of genome-edited crops, clearly stating "that organisms produced by genome editing or by other genetic technologies should not be regulated as GMOs if they could have been produced by traditional breeding methods" ${ }^{\prime}$.

\section{EU legislation does not reflect scientific evidence}

For the past two and a half years, the legal status of genome-edited plants in the EU has been the focus of attention of scientists, breeders and farmers, who are concerned about the impact of the decision of the European Court of Justice (ECJ) of 25 July 2018 in case C-528/16 ${ }^{7}$. The Court held that organisms obtained with techniques of mutagenesis, which were developed since the adoption of the GMO Directive of 2001, are not exempted, even if no foreign DNA is inserted in the genome. Consequently, the risk assessment and traceability, labeling and monitoring obligations provided by the EU GMO directive for the cultivation and commercialization of GMOs apply to crops obtained by genome editing techniques, such as CRISPR. The ECJ's decision is a major setback for European biotechnological research and innovation, as two decades of experience with the market introduction of GMOs in Europe has proven that regulating genome-edited organisms as GMOs blocks the market introduction of genome-edited crops in Europe, in particular for cultivation. Shortly after the ECJ judgment, leading scientists representing European plant

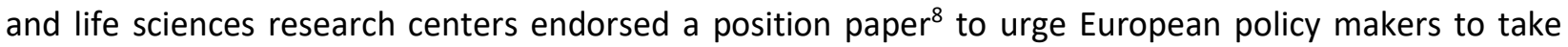
action in order to facilitate the potential of genome editing for agriculture in Europe. Scientists consider the exemption of plant varieties as a result of conventional mutagenesis from the provisions of the European GMO legislation, while not exempting plants of modern, much more targeted approaches of mutagenesis, as a scientifically unjustified discrimination. Moreover, scientific evidence shows that the level of uncertainty about the consequences of the mutagenesis process is much higher with conventional and mutation breeding than with genome editing. The number of off-target mutations resulting from 
genome editing is very low, and undesired traits are easily crossed out in subsequent generations ${ }^{9}$. The features of the plant, rather than the technique used to generate it, should determine its regulatory status. The GMO legislation no longer correctly reflects the current state of scientific knowledge and is, as a consequence, no longer fit for purpose. Furthermore, policy making should be evidence-based, and democratic decision making requires a careful balance between scientific evidence and other aspects, such as societal considerations. However, in the case of genome editing, this balance is distorted by emotionbased opinions. Representing the scientific community, EU-SAGE aims to build awareness of the potential of genome editing in Europe and to urge the European institutions to develop new policies to enable genome editing for more sustainable agriculture and food production. In November 2018, the group of chief scientific advisors to the European Commission published a statement calling for a revision of the GMO legislation in the field of crop improvement, reflecting on the current knowledge and scientific evidence ${ }^{10}$. Since then, besides EU-SAGE, many learned societies, academies of sciences, federations of academies (such as ALLEA and EASAC), biotechnological and other research institutes, as well as the seed and breeding sector (represented by Euroseeds) and the farmer organization (COPA-COGECA, representing 20 million farmers) have taken a similar position. All these recommendations are sciencebased and have the welfare of the European citizens in mind, while there is yet no confirmed scientific fact that argues against the employment of genome editing for precision breeding of crops. Together, scientists reached the highest decision-making organizations and in November 2019, the European Council requested the European Commission to perform a study regarding the status of novel genome techniques under Union law and to submit a proposal if appropriate, including an impact assessment ${ }^{11}$. The scientific community, represented by EU-SAGE, argues that genome edits that do not add foreign DNA to the improved crop should be exempted in the current EU GMO legislation. The outcome of the study from the European Commission will be published and further discussed in 2021, which will be a breaking point to enable the potential of genome-edited plants for more sustainable agriculture in Europe.

From a global perspective, the EU GMO regulatory framework is in complete contrast with other legislative frameworks around the world, which have adopted or are reviewing more 'fit-for-purpose' regulations, as depicted in Figure 1. Four years ago, Argentina was the first country that adopted changes to distinguish the regulatory status of genome-edited plants and GMOs. In a recent report ${ }^{12}$, it is described that these regulatory changes in Argentina have been primarily beneficial to small- and medium-sized enterprises and public R\&D laboratories, and has led to a diversification of crop traits.

The European Union has set ambitious targets in the Farm to Fork strategy ${ }^{13}$ of the Green Deal ${ }^{14}$, with the aim to transition agricultural practices into a more sustainable and resilient food system. By 2030, Europe needs to reduce agriculture's dependency on pesticides by $50 \%$ and fertilizers by $20 \%$ and to reduce its impact on climate change. Now, the European Union is at a decision point to establish how it is going to reach the targets outlined in the Green Deal. Genome editing is a revolutionary tool to introduce the genetic changes specifying desired traits in the genome of plants with high precision and efficiency and has further accelerated the breeding process. Genome editing has the potential to make a critical contribution in the coming years to make food systems more sustainable and more resilient to climate change. The ongoing policy developments in Europe will determine how we will transform our agricultural systems to build a greener future. We would like to encourage you to share your voice and/or to join forces with the EU-SAGE network ${ }^{15}$, and to contribute to advising on effective policies for a more sustainable agriculture. 


\section{Concluding remarks}

Genome editing enables development of desired plant varieties in a faster, more efficient and directed manner, and is being widely adopted in public and private research across the world. However, emotionbased opinions overshadow the potential of genome editing applications in Europe. The EU-SAGE network builds awareness and provides evidence-based solutions through genome editing for the problems faced by agriculture in Europe and worldwide. Let's continue advising together on effective policies for a more sustainable agriculture.

\section{REFERENCES}

1. https://www.nobelprize.org/prizes/ chemistry/2020/press-release/.

\section{2. https://www.eu-sage.eu/.}

3. ALLEA, lead authors: Dima, O., Bocken H., Custers, R., Inzé, D., Puigdomenech, P. (2020). Genome editing for crop improvement. Symposium summary. Berlin, https://doi. org/10.26356/gen-editing-crop.

4. Menz, J., Modrzejewski, D., Hartung, F., Wilhelm, R., and Sprink, T. (2020). Genome edited crops touch the market: a view on the global development and regulatory environment. Front. Plant Sci. 11, 586027.

5. Animal and Plant Health Inspection Service (2020). Regulated article letters of inquiry, https://www.aphis.usda.gov/aphis/ourfocus/biotechnology/am-i-regulated/regulated_article_ letters_of_inquiry/regulated_article_letters_of_inquiry.

6. Department for Environment Food and Rural Affairs (2021). The regulation of genetictechnologies, https://consult.defra.gov.uk/agrifood-chain-directorate/the-regulation-of-genetictechnologies/.

7. Judgment of the Court of Justice of 25 July 2018. Confédération Paysanne and Others v. Premier Ministre and Ministre de L'Agriculture, de l'Agroalimentaire et de la Forêt. Case C-528/16. ECLI:EU:C:2018:583. Available at: https://eur-lex. europa.eu/legal-content/EN/TXT/?qid=159047186 7015\&uri=CELEX:62016CJ05288.

8. https://www.eu-sage.eu/sites/default/ fi les/2020-02/Position\%20paper\%20on\%20 the\%20ECJ\%20ruling.pdf.

9. Modrzejewski, D., Hartung, F., Sprink, T., Krause, D., Kohl, C., and Wilhelm, R. (2019). What is the available evidence for the range of applications of genome-editing as a new tool for plant trait modification and the potential occurrence of associated off-target effects: a systematic map. Environ. Evid. 8, 27.

10. Statement by the Group of Chief Scientific Advisors (2018). A scientific perspective on the regulatory status of products derived from gene editing and the implications for the GMO directive, https://ec.europa.eu/info/sites/info/fi les/2018_11_gcsa_statement_gene_editing_1.pdf.

11. European Union (2019). Council Decision (EU) 2019/1904 of 8 November 2019 requesting the Commission to submit a study in light of the Court of Justice's judgment in Case C-528/16 regarding the status of novel genomic techniques under Union law, and a proposal, if appropriate in view of the outcomes of the study, https://eur-lex. europa.eu/eli/dec/2019/1904/oj. 
12. Whelan, A.I., Gutti, P., and Lema, M.A. (2020). Gene editing regulation and innovation economics. Front. Bioeng. Biotechnol. 8, 303.

13. European Commission (2019). From farm to fork. Our food, our health, our planet, our future, https://ec.europa.eu/info/strategy/ priorities-2019-2024/european-green-deal/ actions-being-takeneu/farm-fork_en.

14. Europeran Commission (2019). A European Green Deal. Striving to be the first climate-neutral continent, https://ec.europa.eu/info/strategy/ priorities-2019-2024/european-green-deal_en.

15. https://www.eu-sage.eu/join. 


\section{Box 1. Genome editing for more sustainable agriculture.}

Genome editing for more sustainable plant production has the potential to:

- $\quad$ Reduce the number of inputs (water, fertilizer, plant protection products) needed to safeguard crop yields, thereby reducing the amount of greenhouse gas emissions associated with these inputs and the environmental pressure caused by plant protection products.

- Increase the output per unit of land, thereby reducing the amount of arable land needed for food production. The spared land should be used for re-wilding and re-forestation.

- Save crop species in danger of disappearance, local varieties and orphan species, thereby protecting biodiversity and increasing the diversification of agricultural species

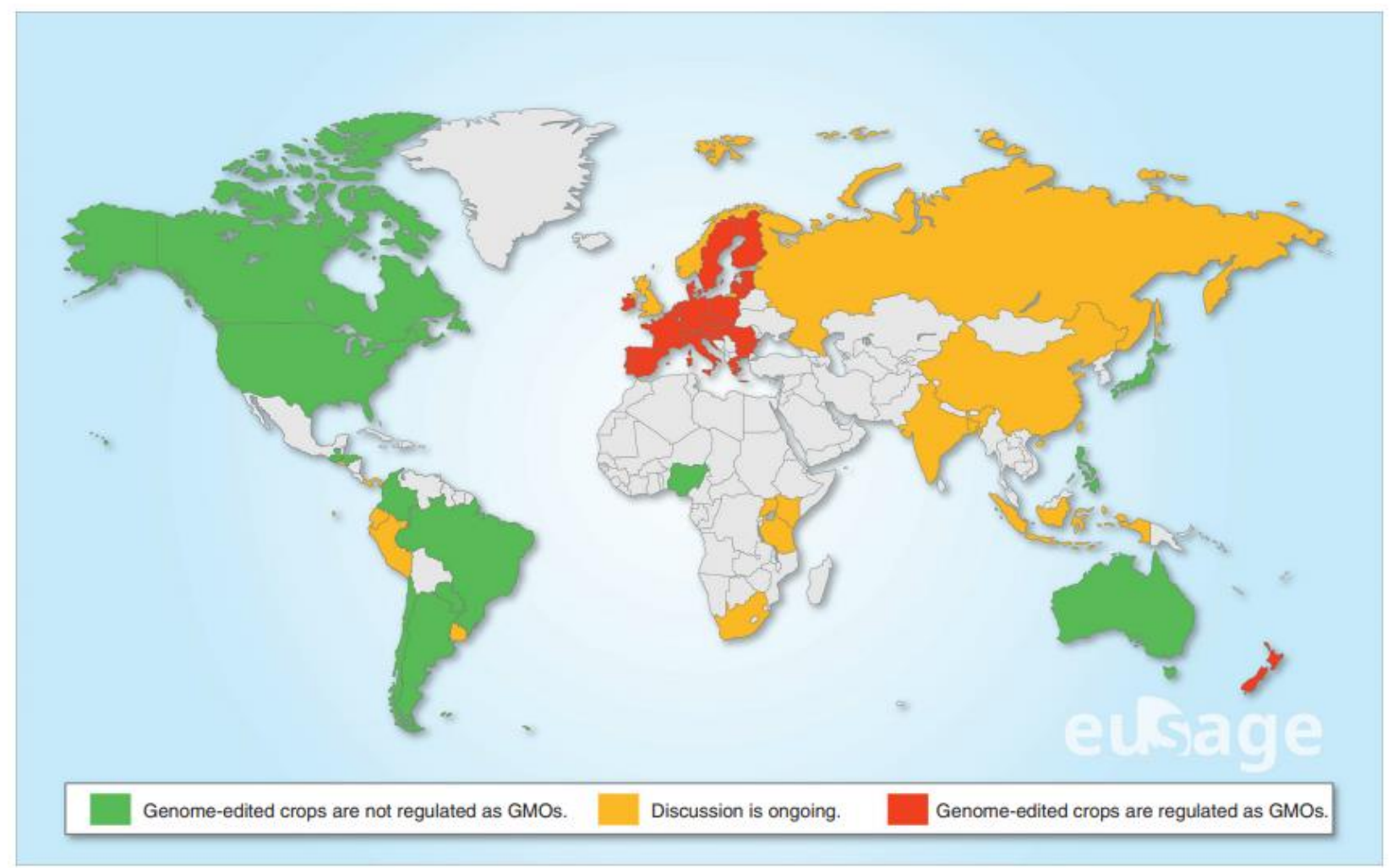

Figure 1. Global overview of regulatory approaches implemented or discussed in different countries for genome-edited crops.

This world map illustrates the current regulatory landscape of genome-edited crops (status February 2021). Genome-edited crops are not regulated as GMOs in green-colored countries, while in yellowcolored countries, legislation to enable the market release of genome-edited crops is being discussed. In the European Union and New Zealand (indicated in red), genome-edited crops are regulated as GMOs. (Graphic courtesy of EU-SAGE.) 